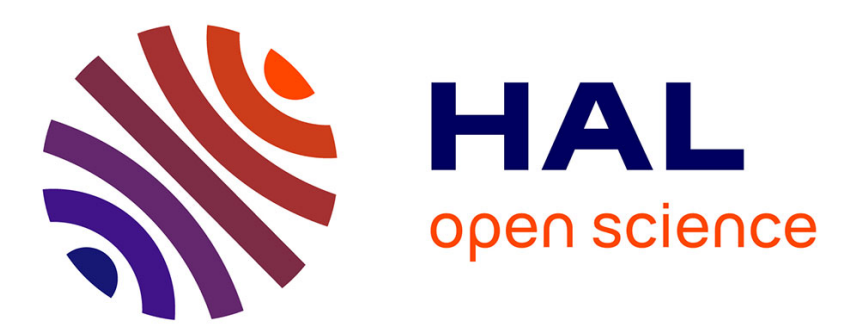

\title{
Cache-enabled Small Cell Networks with Local User Interest Correlation
}

Zheng Chen, Marios Kountouris

\section{To cite this version:}

Zheng Chen, Marios Kountouris. Cache-enabled Small Cell Networks with Local User Interest Correlation. IEEE International Workshop on Signal Processing Advances in Wireless Communications, IEEE, Jun 2015, Stockholm, Sweden. pp.680 - 684, 10.1109/spawc.2015.7227124 • hal-01258025

\section{HAL Id: hal-01258025 \\ https://hal-centralesupelec.archives-ouvertes.fr/hal-01258025}

Submitted on 18 Jan 2016

HAL is a multi-disciplinary open access archive for the deposit and dissemination of scientific research documents, whether they are published or not. The documents may come from teaching and research institutions in France or abroad, or from public or private research centers.
L'archive ouverte pluridisciplinaire HAL, est destinée au dépôt et à la diffusion de documents scientifiques de niveau recherche, publiés ou non, émanant des établissements d'enseignement et de recherche français ou étrangers, des laboratoires publics ou privés. 


\title{
Cache-enabled Small Cell Networks with Local User Interest Correlation
}

\author{
(Invited Paper) \\ Zheng Chen \\ Laboratoire de Signaux et Systèmes (L2S, UMR8506) \\ CentraleSupélec - CNRS - Université Paris-Sud \\ Gif-sur-Yvette, France \\ Email: zheng.chen@centralesupelec.fr \\ Marios Kountouris \\ Mathematical and Algorithmic Sciences Lab \\ France Research Center \\ Huawei Technologies Co. Ltd. \\ Email: marios.kountouris@huawei.com
}

\begin{abstract}
In this paper, we study cache-enabled small cell networks (SCNs) with local regularly requested content sampling to take into account local user interests for the cache decisions. We consider Zipf-like local content popularity with variables indicating the correlation level of user interests in the same region. Based on stochastic spatial models for the small cell base station (SCBS) and user distribution, we provide analytical results on the cache service probability, i.e. the probability that an arbitrary user finds its requested content cached in its nearby SCBSs. The tradeoff between the service probability and the sampling cost is discussed and the optimal sampling range given by maximizing the service probability under the cost constraint is derived. Numerical results with different correlation levels of local user interests are given, which validate our analysis on the service-cost tradeoff.
\end{abstract}

Index Terms - small cell caching, user interest correlation

\section{INTRODUCTION}

Wireless demand for video content has become the dominant source of the exponentially growing data traffic over wireless networks, requiring adequate capacity increase of future wireless networks. Traditional methods to increase network capacity under the conventional macrocell architecture, including more spectrum and improved modulation and coding schemes, are often costly and will not bring sufficient capacity gain to cope with the increasing trend of the wireless data traffic. Network densification with heterogeneous equipment deployment (e.g. pico and femto base stations) has been introduced as an expansion to existing macrocell networks to improve network throughput and spatial reuse of communication resources. Such low-power and short-range small base stations can not only reduce traffic load on the macro base stations (MBSs), but also serve mobile users in small cells with lower latency.

Nevertheless, in small cell networks (SCNs), the backhaul capacity becomes the performance and cost bottleneck. Deploying high-speed optical fiber backhaul to connect small cell access points to the core network may not be an economically viable option. To face this challenge, small cell caching has emerged as one of the solutions for the backhaul bottleneck, which exploits the fact that the content is brought closer to end-users before being requested. The basic idea of cacheenabled SCNs is to integrate large-capacity storage units at small cell base stations (SCBSs). Popular video content can be stored automatically in the cache of SCBSs at off-peak hours, then serve user requests during peak hours. When a local user requests for a file already cached in its covering SCBS, the service latency is largely reduced since it does not have to pass through the backhaul to retrieve the content from remote servers. Another benefit of small cell caching is the exploitation of the high degree of asynchronous content reuse of wireless video demand, where repeated video content is requested by different users asynchronously. Caching at small cells can avoid repeated transmission of the same video content from the core network to end-users, especially in the case where local users share similar interest in the requested content.

The literature of cache-enabled wireless networks concerns many aspects of caching problems under the structure of advanced wireless networks, e.g. cacher distribution, content placement, caching and replacement policy. FemtoCaching networks with distributed caching helpers are studied in [1]. The optimal file assignment to minimize the expected downloading time under the uncoded and coded caching cases is analyzed. In [2] a small cell caching problem is considered using tools from stochastic geometry for the network modeling, with analytical results given on some basic performance metrics and fundamental tradeoffs. Prior studies investigating wireless caching problems often adopt Zipf distribution as a referential model for the global popularity distribution of requested content and assumes similar popularity pattern for all users in the system. Under the consideration of different interest levels over requested content, a clustering algorithm is proposed in [3] to group users into clusters based on their request profile. Optimal caching decisions are then made at each SCBS by using a distributed regret learning approach to minimize the total service delay.

In this paper, we consider that caching decisions are made at SCBSs based on local users' request pattern rather than the global content popularity, as in cache-enabled SCNs each SCBS only serves a small amount of users. Specifically, each SCBS constructs its local regular content library by sampling regularly requested files of users in its sampling range, then determines the set of files to be cached under limited cache 
capacity. We choose the conventional "cache the most popular files" strategy to give baseline analysis on the impact of user interest correlation on the cache hit/miss probability. The results can be generalized to cases with more advanced caching strategies as proposed in [4] and [5]. We assume Zipf-like distribution for the local regular content popularity in the same region. Under stochastic spatial models for the SCBS locations and user distribution, we derive the cache service probability as a function of the maximum sampling distance. We also consider that there is a sampling cost, which is modeled as a distance-dependent function. The tradeoff between the cache service probability and the sampling cost is also studied, and the optimal sampling distance subject to a cost constraint is provided.

\section{Network Modeling AND ANALYSIS}

\section{A. Network Model and Basic Assumptions}

We consider a cache-enabled SCN model with limited storage space at each SCBS, serving its nearby users within a certain distance according to its transmit power constraint. We model the SCBS distribution on the two-dimensional Euclidean plane $\mathbb{R}^{2}$ by a homogeneous PPP $\Phi_{c}=\left\{Y_{i}, i \in \mathbb{N}^{+}\right\}$ with intensity $\lambda_{c}$, where $Y_{i}$ denotes the position of the $i$-th SCBS. Users to be served are distributed according to another independent homogeneous $\operatorname{PPP} \Phi_{u}=\left\{x_{j}, j \in \mathbb{N}^{+}\right\}$with intensity $\lambda_{u}$, where $x_{j}$ denotes the position of the $j$-th user.

We introduce the notion of "local user interest pattern", which can be acquired when allowing each SCBS to sample the regularly requested content of all users in its sampling region of radius $R_{p}$. The small cell cachers decide which files to cache based on the adopted caching policy. Assume there exists a maximum distance that a SCBS can serve, denoted by $R_{v}$, the disk centered at the SCBS with radius $R_{v}$ can be seen as the service region of this SCBS.

When increasing the sampling range, the SCBSs may have better knowledge of local user interest pattern, but due to the limited cache storage only the most popular files will be stored. Then, for a random user being sampled the probability of finding its regular content cached at its covering SCBSs will decrease with the sampling region size. The service range constraint also gives un upper bound on the probability of an arbitrary user being served by the small cell cachers. We denote this service probability by $P_{s v}$. Increasing the sampling range also leads to higher cost of the local request pattern learning at the SCBSs. In this paper we focus on the influence of the sampling range on the service probability and the sampling cost for different levels of local interest correlation among small cell users.

Suppose that each user has a library of size $J$, which contains its regularly requested files. For simplicity, we assume that all the files have equal unit size. Each SCBS has limited cache storage size denote by $M$, which is the maximum number of files that can be stored. Denote by $N$ the number of users in the sampling region of a SCBS, from point process theory, we have $\mathbb{E}[N]=\lambda_{u} \pi R_{p}^{2}[6]$.
When users in the same sampling region share similar interest in requested content, different users may have overlapped files in their regular content libraries, so the overall regular content library size will not grow linearly with the number of users in this area. Conditioning on having $N$ users in the sampling region of a typical SCBS, we denote by $\mathcal{C}=\left\{c_{1}, \ldots, c_{S}\right\}$ the local content library of the $N$ users with $S$ representing the library size. Denote by $g: \mathbb{N}^{+} \rightarrow \mathbb{N}^{+}$ the mapping function from the number of users $N$ to the local library size $S$. In reality $S=g(N)$ can be learned numerically at the SCBSs and the approximate function can be found by data fitting. Here we assume that $g$ is a piecewise function and follows $S=\min (J N,\lceil J(1+\mu \log N)])$, where $\mu$ is a constant factor that characterizes the similarity level of local user interest.

For a given local regular content library, we assume that the popularity distribution of files in it follows the ZipfMandelbrot law, that is, for the $i$-th most popular file we have its request probability as

$$
p_{i}=\frac{\Omega}{(q+i)^{\gamma}}
$$

where $\Omega=\left(\sum_{n=1}^{S}(q+n)^{\gamma}\right)^{-1}$ is the normalization constant, $q$ is the shift parameter which is related to the shifting based on the Zipf distribution, and $\gamma$ is the shape parameter, which defines the concentration (correlation) level of the content popularity. Similarly to the $g$ function, the real popularity distribution can be learned by sampling and sorting the popularity of all requested files in the local region.

Note that $\mu$ in the $g$ function defines how similar are the regular content libraries of users in the same area, $\gamma$ in the popularity distribution defines the relative disparity of content popularity in the local library. The combination of these two factors gives full characterization of the correlation level of local user interest.

\section{B. Service Probability Analysis}

We consider at first a baseline caching policy, which is "cache the most popular files". Cache entities at the SCBSs have identical and limited storage size as $M$ files. Assume that we have a SCBS at the origin with $N$ users in its sampling region of radius $R_{p}$. The local content library size is known as $S$. When $M \geq S$, all files in the local content library can be stored at the SCBS. When $M<S$, due to the limited cache storage only the most popular $M$ files in the sampling region can be cached. The cache miss probability $P_{m}$, which is the probability that a random file in the local regular content library $\mathcal{C}$ not being cached, can be given as

$$
P_{m}=\sum_{i=M+1}^{S} p_{i}=\sum_{i=M+1}^{S} \frac{1}{(q+i)^{\gamma} \sum_{n=1}^{S}(q+n)^{\gamma}} .
$$

Evidently, $P_{m}$ increases with $S$, which verifies the intuition that larger content library size leads to higher cache miss probability when the cache storage capacity is limited. Assume 
that users outside the sampling range of a SCBS have almost negligible probability to find their regularly requested content in this small cell cache. The service probability $P_{s v}$ is then denoted by the probability of the intersection of three events:

- $\mathcal{E}_{1}=$ user's regular content library being sampled by at least one SCBS,

- $\mathcal{E}_{2}=$ the requested content being cached,

- $\mathcal{E}_{3}=$ user in the service range of the sampling SCBS.

Furthermore we have $\mathcal{E}_{1} \subseteq \mathcal{E}_{3}$ if $R_{p} \leq R_{v}$ and $\mathcal{E}_{3} \subset \mathcal{E}_{1}$ otherwise. In the case where $R_{p} \leq R_{v}, P_{s v}$ can be given by

$$
\begin{aligned}
P_{s v}= & \mathbb{P}[\text { user being sampled } \cap \text { content being cached }] \\
= & 1-\mathbb{P}[\text { not being sampled }] \\
& -\sum_{k=1}^{\infty} \mathbb{P}[\text { sampled by } \mathrm{k} \text { cachers }] \cdot \mathbb{P}[\mathrm{k} \text { cache miss }] \\
= & 1-e^{-\lambda_{c} \pi R_{p}^{2}} \\
& -\sum_{k=1}^{\infty} \frac{\left(\lambda_{c} \pi R_{p}^{2}\right)^{k}}{k !} \exp \left(-\lambda_{c} \pi R_{p}^{2}\right) \prod_{j=1}^{k} P_{m}^{j}
\end{aligned}
$$

where $P_{m}^{j}$ is the cache miss probability of the $j$-th SCBS which has the typical user within its sampling range. When different small cells have different request correlation levels, which corresponds to different $\gamma$ in the Zipf distribution, the cache miss probability can be given as

$$
P_{m}^{j}=\sum_{i=M+1}^{S} \frac{1}{(q+i)^{\gamma_{j}} \sum_{n=1}^{S}(q+n)^{\gamma_{j}}}
$$

where $\gamma_{j}$ is the shape parameter of the content popularity distribution in the sampling region of the $j$-th SCBS. It is easy to see that $P_{s v}$ increases monotonically with $R_{p}$, meaning that larger sampling distance gives higher probability of a random user to find its regularly requested content cached in the covering SCBSs.

In the case where $R_{p}>R_{v}$, the service probability is given by

$$
\begin{aligned}
P_{s v}= & \mathbb{P}[\text { user in service range } \cap \text { content being cached }] \\
= & 1-e^{-\lambda_{c} \pi R_{v}^{2}} \\
& -\sum_{k=1}^{\infty} \frac{\left(\lambda_{c} \pi R_{v}^{2}\right)^{k}}{k !} \exp \left(-\lambda_{c} \pi R_{v}^{2}\right) \prod_{j=1}^{k} P_{m}^{j} .
\end{aligned}
$$

Contrary to the previous case, for fixed $R_{v}$ the service probability decreases with $R_{p}$, which is easy to understand since larger sampling region corresponds to higher cache miss probability for users being sampled, while the number of users that can be served will not increase with the sampling range due to the service range constraint. Combining these two cases we have

$$
\begin{aligned}
P_{s v}= & \mathbb{P}[\text { user in service range } \cap \text { content being cached }] \\
= & 1-e^{-\lambda_{c} \pi \min \left\{R_{p}, R_{v}\right\}^{2}}-\sum_{k=1}^{\infty} e^{-\lambda_{c} \pi \min \left\{R_{p}, R_{v}\right\}^{2}} . \\
& \frac{\left(\lambda_{c} \pi \min \left\{R_{p}, R_{v}\right\}^{2}\right)^{k}}{k !} \prod_{j=1}^{k} P_{m}^{j} .
\end{aligned}
$$

From the above result we see that for a given constraint on the service range of a SCBS, the service probability increases with $R_{p}$ till it reaches the service range limit $R_{v}$, then it decreases monotonically with $R_{p}$.

\section{Sampling Cost Analysis}

The regular content sampling allows the SCBSs to learn the interests of local users, thus the service offered by small cell cachers can be better adapted to the local request pattern. On the other hand, the power consumption for the sampling procedure must be taken into consideration for the purpose of energy efficiency. Denote by $C_{s}$ the sampling cost of the typical SCBS at the origin with sampling region radius $R_{p}$, we introduce a cost function for the sampling process, which depends on the distance from the sampled users to the SCBS, i.e.

$$
C_{s}=\sum_{j \in \Phi_{u} \cap \mathcal{B}\left(0, R_{p}\right)} f\left(\left\|x_{j}-Y_{0}\right\|\right) \cdot \eta
$$

where $\left\|x_{j}-Y_{0}\right\|$ is the Euclidean distance between the $j$-th user in the sampling region and the typical SCBS at the origin, $f$ is a distance-dependent cost function, $\eta$ is a constant cost factor. For simplicity we choose $\eta=1$ and inverse pathloss model for $f$ as $f(x)=x^{\alpha}$ where $\alpha$ is the pathloss exponent.

From Campbell's theorem, we have the mean cost when averaging over different realizations of PPP as

$$
\begin{aligned}
\mathbb{E}\left[C_{s}\right] & =\mathbb{E}\left[\sum_{j \in \Phi_{u} \cap \mathcal{B}\left(0, R_{p}\right)}\left\|x_{j}-Y_{0}\right\|^{\alpha}\right] \\
& =2 \pi \lambda_{u} \int_{0}^{R_{p}} r^{\alpha} \cdot r \mathrm{~d} r \\
& =\frac{2 \pi \lambda_{u}}{\alpha+2}\left(R_{p}^{\alpha+2}-1\right) .
\end{aligned}
$$

We see that the sampling cost increases linearly with the user density and follows power law of order $\alpha+2$ growth with the sampling region radius. Note that in dense network deployments, larger sampling distance results in improved learning of the request pattern; however the sampling cost may be prohibitively high and should be taken into account when designing the optimal sampling distance.

\section{Optimal SAmpling RANGE UNDER COST CONSTRAINT}

In Section II we gave basic results on the service probability and sampling cost for cache-enabled small cell networks with local request pattern learning. Based on our assumptions on 


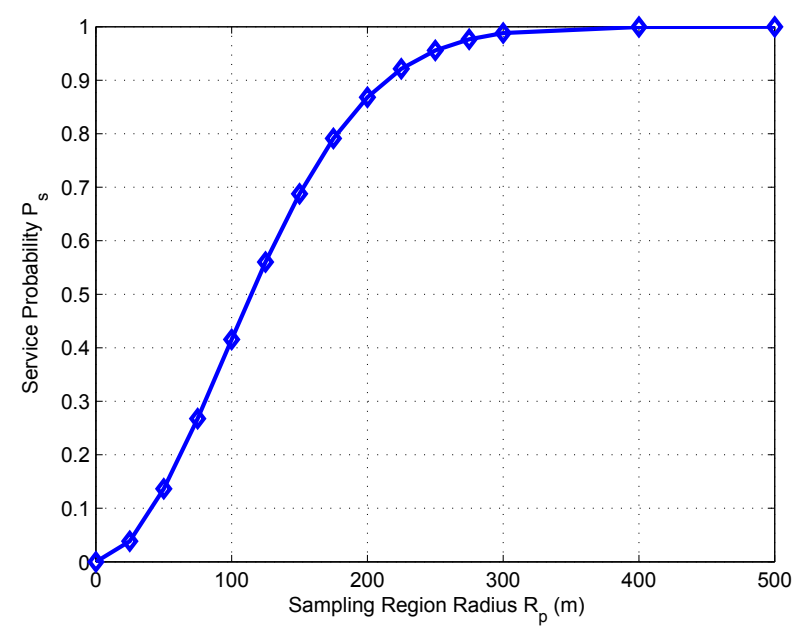

Fig. 1. Service probability vs. sampling range with infinite service range. $q=0, \gamma=1$.

user interest correlation, we can gain insight on how the service probability and cost scale with the sampling range.

Fig. 1 shows an example of the service probability as a function of the sampling region radius when there are no constraint on the service range. We see that increasing sampling range gives higher service probability, but the increment tends to saturate when $R_{v}$ is relatively large. By taking into consideration the limited service range while ignoring the sampling cost, the optimal sampling region size is equal to $R_{v}$ since it gives the highest service probability, as we have analyzed in Section II. In this section, we search the optimal sampling range which gives sufficiently good service probability while assuring the sampling cost is below a given constraint.

We formulate the problem as follow:

$$
R_{p}^{*}=\arg \max _{R_{p}} P_{s v}
$$

Subject to:

$$
\begin{aligned}
& R_{p} \in\left[0, R_{v}\right], \\
& C_{s}\left(R_{p}^{*}\right)<C_{\max },
\end{aligned}
$$

$C_{\text {max }}$ is the predefined cost constraint. The first condition comes from the fact that when $R_{p}>R_{v}$ the service probability decreases monotonically with $R_{p}$. The second condition is due to the sampling cost constraint.

With the help of (8), we can derive the constraint on $R_{p}$ to satisfy (11) as

$$
R_{p}^{*}<\left(\frac{C_{\max }(\alpha+2)}{2 \pi \lambda_{u}}+1\right)^{\frac{1}{\alpha+2}} .
$$

Since $P_{s v}$ is a monotonically increasing function of $R_{p}$ for $R_{p} \in\left[0, R_{v}\right]$, combining these two constraints we have the optimal sampling distance given by $R_{p}^{*}=$ $\min \left(R_{v},\left(\frac{C_{\max }(\alpha+2)}{2 \pi \lambda_{u}}+1\right)^{\frac{1}{\alpha+2}}\right)$.

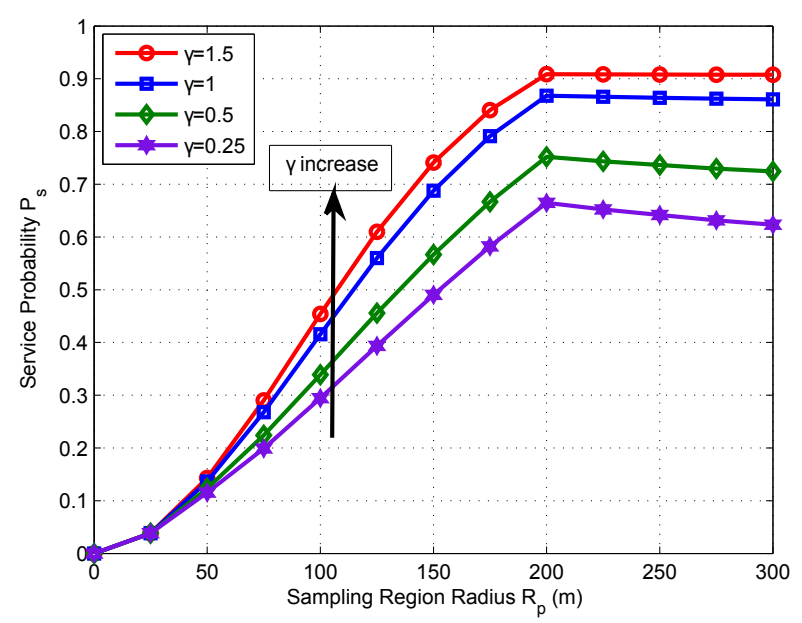

Fig. 2. Service probability under different levels of user interest correlation.

\section{NumERICAL RESUlts}

In this section we validate our analysis on cache-enabled small cell networks with local user interest sampling. We show the tradeoff between the small cell cache service probability and the sampling cost for different levels of local user interest correlation and user densities.

We set $\lambda_{c}=2 \times 10^{-5}$ and $\lambda_{u}=8 \times 10^{-4}$ as the density of SCBSs and of users to be served. The service region of each SCBS has radius $R_{v}=200 \mathrm{~m}$. The regular content library size of each user is $J=10$. The maximum cache storage of a SCBS is $M=50$. We use $S=\min (J N,\lceil J(1+3 \log N)\rceil)$ as the mapping function from the number of users to the overall local content library size. The popularity distribution is assumed to follow the Zipf-Mandelbrot law with $q=0$ (Zipf's law). We assume the same shape parameter for the content popularity distribution in the sampling region of all the SCBSs, meaning that $\gamma_{j}=\gamma$ for $j=1, \cdots, \infty$. Note that considering uniform or different shape parameters for the popularity distribution does not change the general trend of how the sampling distance affects the service probability $P_{s v}$. Numerical results are presented with different values of the shape parameter $\gamma \in[0.25,1.5]$, which correspond to different user interest correlation levels, in order to see how the cache service performance scales with local user interest pattern.

\section{A. Service Probability under Different Correlation Levels of Users' Interest}

In Section II, we saw that the cache service performance depends on the local user interest correlation level. In Fig. 2 we plot the service probability $P_{s v}$ as a function of the sampling range $R_{p}$ for $\gamma=0.25,0.5,1$ and 1.5 respectively.

From Fig. 2, we see that $P_{s v}$ increases with $R_{p}$ before it reaches $R_{v}=200 \mathrm{~m}$ then decreases when $R_{p}$ continues to grow. However, the speed of decrease is relatively small compared to the growth in $R_{p} \in\left[0, R_{v}\right]$, especially for higher values of $\gamma$. This is because higher $\gamma$ corresponds to higher concentration of content popularity. Though increasing library 


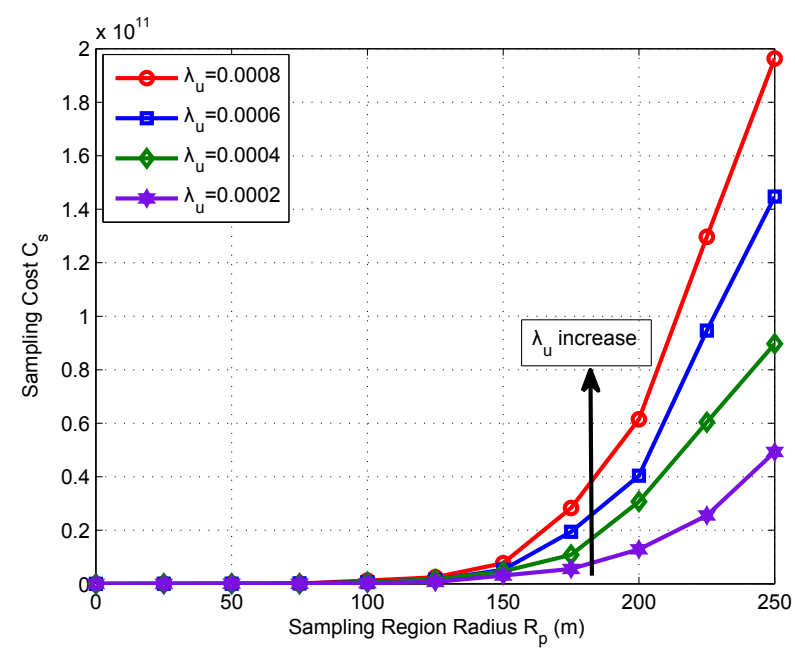

Fig. 3. Sampling cost vs. sampling range with distance-dependent cost function.

size increases the number of files not being cached, the most popular files are cached with higher probability, which still leads to lower cache miss probability in the sampling region.

\section{B. Sampling Cost}

Fig. 3 shows the simulated sampling cost per SCBS as a function of the maximum sampling distance with the adopted distance-dependent cost function. The simulation results are obtained under different user density settings and by averaging over 5000 PPP realizations. It shows the dramatic growth of the cost of local regular content sampling when increasing the sampling distance $R_{p}$. In a high user density scenario, the cost issue is of growing importance, showing the need for taking into consideration the sampling cost when searching for the optimal sampling distance.

\section{CONCLUSIONS}

In this paper we investigated cache-enabled SCNs with local user interest sampling managed by SCBSs for local request pattern learning. We provided analytical and numerical results on the cache service performance and the sampling cost, as well as the optimal sampling distance to maximize the cache service probability under a given cost constraint. The main takeaway of this paper is the idea of local regular content sampling and the influence of the sampling distance on the cache service performance, which will be beneficial for designing the sampling procedure of cache-enabled SCNs.

\section{REFERENCES}

[1] N. Golrezaei, K. Shanmugam, A. Dimakis, A. Molisch, and G. Caire, "Femtocaching: Wireless video content delivery through distributed caching helpers," in Proceedings IEEE INFOCOM, March 2012, pp. $1107-1115$.

[2] E. Bastug, M. Bennis, and M. Debbah, "Cache-enabled small cell networks: Modeling and tradeoffs," in IEEE International Symposium on Wireless Communications Systems (ISWCS), Aug 2014, pp. 649-653.
[3] M. S. ElBamby, M. Bennis, W. Saad, and M. Latva-aho, "Content-aware user clustering and caching in wireless small cell networks," in IEEE International Symposium on Wireless Communications Systems (ISWCS), Aug 2014, pp. 945-949.

[4] B. Blaszczyszyn and A. Giovanidis, "Optimal geographic caching in cellular networks," in IEEE International Conference on Communications (ICC), Jun 2015.

[5] M. Ji, G. Caire, and A. F. Molisch, "Wireless device-to-device caching networks: Basic principles and system performance," CoRR, vol abs/1305.5216, 2013. [Online]. Available: http://arxiv.org/abs/1305.5216

[6] A. Baddeley, Spatial Point Processes and their Applications, Springer Verlag, 2007, vol. 1892, pp. 1-75. 